\title{
Dopants in Semiconductors Studied by Perturbed Angular Correlation
}

\author{
J. Bartels, K. Lorenz, F. Ruske, G. Tessema \\ AND R. VIANDEN \\ Institut für Strahlen- und Kernphysik der Universität Bonn \\ 53115 Bonn, Germany
}

\begin{abstract}
The $\gamma-\gamma$ perturbed angular correlation technique is a very powerful tool for the investigation of dopant incorporation and damage recovery after implantation in semiconductors. The basic principles of the technique will be introduced followed by a discussion of its strengths and limitations. Examples of its application will be given, ranging from cavities in silicon, effects of uniaxial stress on acceptor-donor pairs in silicon to damage recovery in nitride semiconductors like GaN.
\end{abstract}

PACS numbers: $61.72 . \mathrm{Vv}, 61.72 . \mathrm{Tt}, 81.40 . \mathrm{Ef}, 76.80 .+\mathrm{y}$

\section{Introduction}

Materials properties can be modified in a wide range by introducing defects in a controlled fashion. This is a well-known technique which has been employed from the times when man first used metallic tools up to today, when defects, e.g. dopants in semiconductors, are essential for the ubiquitous integrated circuits which play an essential role in our civilisation.

Defect is a general term describing any deviation from the perfect structure in crystalline materials. The most simple defects (so-called point defects) can either be vacant lattice sites (vacancies), regular lattice atoms displaced from regular sites (interstitials) or in compound crystals atoms on the site of the other constituent (antisites). Further, impurities incorporated on substitutional or interstitial sites are generally regarded as point defects.

Often defects influence the properties of the base material very effectively. Therefore, any method to study these defects must be sensitive enough to detect and investigate defects at concentration levels ranging from a few percent down to about $10^{-10} \mathrm{~cm}^{-3}$ volume concentration in semiconductors. 
Hyperfine interaction techniques have successfully contributed to our knowledge of materials properties [1] and the nature and behaviour of defects in metals [2] and semiconductors [3]. Due to its short-sightedness the various techniques measuring the hyperfine interaction are particularly sensitive to the immediate vicinity of the probe atom and thus in many cases a microscopic picture of the probe-defect complex can be deduced.

Among the various methods capable of measuring the influence of the hyperfine interaction on the atomic nucleus only the perturbed angular correlation (PAC) method, which employs radioactive probe atoms, will be treated here. The reason is that classical methods like nuclear magnetic resonance, nuclear quadrupole resonance or measurements of the nuclear specific heat are only applicable if the nuclei under study are quite abundant in the sample under study and are therefore less useful for the study of highly diluted dopants and defects.

\section{Hyperfine interaction}

Historically the term hyperfine interaction was used to describe the interaction of the atomic nucleus with the electrons of its own shell [4]. Nowadays, however, the term is generally used to describe the interaction between the atomic nucleus and the electromagnetic field built up by the atomic electrons, the electrons and atoms of the crystal and/or external sources. The basic theory of the hyperfine interaction is well developed and excellent and complete presentations are available in the literature $[5,6]$. Therefore, only a brief introduction to the basic ideas and notation will be given here.

Generally, the Hamiltonian describing the hyperfine interaction consists of a magnetic and an electric part

$$
\boldsymbol{H}=\boldsymbol{H}_{\mathrm{mag}}+\boldsymbol{H}_{\mathrm{el}} .
$$

The first part

$$
\boldsymbol{H}_{\mathrm{mag}}=-g \mu_{\mathrm{N}} \boldsymbol{H} \cdot \boldsymbol{I}
$$

describes the magnetic interaction of a nucleus of spin $\boldsymbol{I}$ and nuclear magnetic Landé factor $g$ with a magnetic field $H$ ( $\mu_{\mathrm{N}}$ - nuclear magneton). Since in most semiconductors no internal magnetic field is present, the interaction with external magnetic fields is used only to clear up ambiguities about the quadrupole interaction caused by a defect.

The second part, $\boldsymbol{H}_{\mathrm{el}}$, is due to the electrostatic potential energy of a nucleus of finite dimensions in the field of the surrounding charges. Under the assumption that the nuclear radius is small compared to the distance to the field generating charges, the potential can be expanded in a multipole series about the centre of the nucleus. In the resulting series the first term represents simply the Coulomb energy of a point charge $Z$ ( $Z$ - atomic number of the nucleus) in the field. Since it is independent of the $m$ quantum number of the sublevels, i.e., the orientation 
of the nucleus, it causes only a general energy shift of all sublevels and is of no relevance to the further considerations. The second term, the dipole term, vanishes, since, due to parity conservation, nuclei have no observable electric dipole moment. The first non-vanishing term is therefore the quadrupole term $\boldsymbol{H}_{Q}$, which can be expressed as the inner product of two second rank Cartesian tensors

$$
\boldsymbol{H}_{\mathrm{el}} \cong \boldsymbol{H}_{Q}=\sum_{i j} Q_{i j} V_{i j}
$$

The nuclear quadrupole moment operator $Q_{i j}$ is defined by the integral over the nuclear charge density $\Delta_{N}(r)$

$$
Q_{i j} \equiv \int \rho_{N}(r)\left(3 \chi_{i} \chi_{j}-r^{2} \delta_{i j}\right) \mathrm{d} r
$$

or can be written in terms of the nuclear spin operators as

$$
Q_{i j}=\frac{e Q}{6 I(2 I-1)}\left[\frac{3}{2}\left(I_{i} I_{j}+I_{j} I_{i}\right)-\delta_{i j} I(I+1)\right],
$$

where $Q$ is the quantity conventionally referred to as the "nuclear quadrupole moment". $Q$ is measured in barn $\left[1\right.$ barn $\left.=10^{-24} \mathrm{~cm}^{2}\right]$ and values of a few tenths of a barn in nearly spherical nuclei to several barns in highly deformed nuclei are found. A recent tabulation of quadrupole moments is given by Raghavan [7].

The electric field gradient (EFG) tensor $V_{i j}$ is defined as the second spatial derivative of a classical electrostatic potential $V$ evaluated at the nuclear site

$$
V_{i j} \equiv \frac{\partial^{2} V}{\partial x_{i} \partial x_{j}}
$$

$V_{i j}$ is a symmetric second rank tensor. Further, if electrons with a spherically symmetric charge distribution, i.e., electrons in $s$ and $p_{1 / 2}$ states, which produce no field gradient at the nuclear site are excluded, it can be derived from the Laplace equation $\Delta V=0$ that $V_{i j}$ is traceless. Such a second rank tensor can always be diagonalized by a coordinate transformation to a principal axis system. Conventionally, the principal axes are chosen such that $V_{z z} \geq V_{y y} \geq V_{x x}$, and $V_{i j}$ can be described completely by the so-called principal component $V_{z z}$ and the asymmetry parameter $\eta$

$$
\eta \equiv \frac{V_{x x}-V_{y y}}{V_{z z}}
$$

Regarding the effect of the symmetry of the environment on $V_{i j}$, one first notes that in the event of cubic point symmetry about the nuclear site, all three coordinate directions $x, y$, and $z$ are equivalent. In this case the traceless property of $V_{i j}$ then requires all components of $V_{i j}$ to be zero and no interaction can be observed. In the case of axial symmetry the $x$ and $y$ directions are equivalent and thus, according to Eq. (7), the asymmetry parameter $\eta$ will become 0 . Should the point symmetry be rhombic or lower $V_{x x}$ will differ from $V_{y y}$ and $\eta$ assumes values between 0 and 1 . 
$V_{z z}$ is usually given in units of $\mathrm{V} / \mathrm{cm}^{2}$. Experimental values for $V_{z z}$ are in the range of $10^{17} \mathrm{~V} / \mathrm{cm}^{2}$ in metals [1] and can reach values around $10^{18} \mathrm{~V} / \mathrm{cm}^{2}$ in compounds [8].

The interaction energy between the nuclear quadrupole moment and the electric field gradient is given by the Hamilton operator

$$
\boldsymbol{H}_{Q}=\frac{e Q V_{z z}}{4 I(2 I-1)}\left[3 I_{z}^{2}-I(I+1)+\frac{\eta}{2}\left(I_{x}^{2}+I_{y}^{2}\right)\right]
$$

with the angular momentum operators $\boldsymbol{I}_{z}, \boldsymbol{I}_{x}$, and $\boldsymbol{I}_{y}$.

In order to obtain the energy eigenvalues of $\boldsymbol{H}_{Q}$ generally a numerical diagonalization is necessary. Only in the special case of an axially symmetric electric field gradient $(\eta=0)$ the Hamiltonian becomes diagonal in a representation with the principal axis $z$ as the axis of quantization. The energy eigenvalue of a nuclear state with spin $I$ and $z$ component $m$ is then given by

$$
E_{m}=\frac{e Q V_{z z}}{4 I(2 I-1)}\left[3 m^{2}-I(I+1)\right]
$$

and the splitting between two sublevels $m$ and $m^{\prime}$ becomes

$$
E_{m}-E_{m^{\prime}}=\frac{e Q V_{z z}}{4 I(2 I-1)} 3\left|m^{2}-m^{\prime 2}\right| \text {. }
$$

From Eq. (9) it becomes clear that unlike the case of magnetic interaction the quadrupole interaction splitting of a nuclear level depends on its spin $I$ and that the + and $-m$ states remain degenerate.

For a nuclear state with $I=5 / 2 \mathrm{Eq}$. (10) yields the following transition frequencies between the $m$ substates which are conventionally expressed as multiples of the so-called fundamental quadrupole frequency

$$
\omega_{Q}=\frac{e Q V_{z z}}{4 I(2 I-1) \hbar},
$$

$\omega_{1}=6 \omega_{Q}, \omega_{2}=12 \omega_{Q}$, and $\omega_{3}=18 \omega_{Q}$. In order to make comparisons easier in the literature often the quadrupole interaction frequency is given

$$
\nu_{Q} \equiv \frac{\omega_{Q} 4 I(2 I-1)}{2 \pi}=\frac{e Q V_{z z}}{h} .
$$

\section{Perturbed angular correlation}

The perturbed angular correlation (PAC) method is based on the fact that the probability of photon emission from an excited nuclear state depends on the angle between the emission direction and the nuclear spin $I$. Since normally the spins of an ensemble of nuclei show no preferred direction, the emission of $\gamma$-rays from a radioactive sample will normally be isotropic. However, this isotropy can be destroyed for nuclei emitting successively two $\gamma$-rays in their decay. By placing a 
detector for the first photon $\gamma_{1}$ in an arbitrary direction one will preferably detect $\gamma_{1}$ from nuclei whose spins are oriented in that direction. The emission probability of $\gamma_{2}$ from each of those nuclei will then be anisotropic with respect to the emission direction of its $\gamma_{1}$, i.e. the $m$ substates of the intermediate state with respect to the quantization axis defined by this direction will be populated unequally.

However, this anisotropy will only stay constant as long as the unequal population of the intermediate substates is preserved during the time the nucleus exists in the intermediate state.

A hyperfine interaction of the nucleus in the intermediate state with extranuclear fields, like an electric quadrupole interaction (QI), as described above, induces periodic changes of the $m$ substate population, unless $V_{z z}$ is collinear with the quantization axis defined by the emission of $\gamma_{1}$. Since this effect causes a perturbation of the original angular correlation the method has been named perturbed angular correlation method.

The changes are equivalent to a precession of the associated radiation pattern about the $z$-axis and lead to a time dependent modulation of the coincidence count rate

$$
N(t)=\exp (-\lambda t) \sum_{n=0}^{n_{\max }} s_{n}\left(\eta, \alpha_{i}\right) \cos \left(c_{n}(\eta) \nu_{Q} t\right)
$$

with $n_{\max }$ being determined by the spins of the nuclear levels and multipolarities of the radiative transitions involved in the $\gamma-\gamma$ cascade. The leading factor describes the exponentially decreasing probability to register $\gamma_{2}$ after the intermediate state with decay constant $\lambda$ has been populated by $\gamma_{1}$. Since $\lambda$ usually is accurately known this exponential factor can be eliminated and only the time dependent variation of the anisotropy due to the nuclear precession is analyzed. As indicated in Eq. (13), the frequency factors $c_{n}$ depend only on the asymmetry of the EFG and can be calculated for all values of $\eta$. Therefore, the $c_{n}$ derived from a Fourier analysis of the observed interaction pattern $N(t)$ allow an accurate determination of the asymmetry parameter $\eta$ of the EFG. The coefficients $s_{n}\left(\eta, \alpha_{i}\right)$ also depend on the orientation of the principal axis system of the EFG (described by the Euler angles $\alpha_{i}$ ) relative to the $z$ axis defined by the emission of $\gamma_{1}$. PAC measurements in oriented single crystals can therefore be used to determine the orientation of the EFGs principal axis system relative to the crystallographic directions of the lattice.

The presently available PAC measuring equipment has reached a considerable degree of sophistication and efficiency. Typical set-ups consist of four fixed scintillation detectors or two detectors, with a third one being moved in regular time intervals between a $90^{\circ}$ and $180^{\circ}$ position relative to a fixed one. A survey of the specific advantages and disadvantages of different set-ups has been given by Arends et al. [9].

As scintillators now usually $\mathrm{BaF}_{2}$ scintillators are employed [10]. Its scintillation light consists of two components one in the UV region with an extremely 
TABLE

The most common PAC probe nuclei and their relevant properties. If not indicated otherwise, the data are taken from Ref. [7].

\begin{tabular}{c|c|c|c|c|c|c|c}
\hline \hline Probe & Parent & $\begin{array}{c}\text { Half-life } \\
\text { of parent } \\
t_{1 / 2}\end{array}$ & $\begin{array}{c}\text { Level } \\
\text { energy } \\
{[\mathrm{keV}]}\end{array}$ & $\begin{array}{c}\text { Half-life } \\
\text { of level } \\
t_{1 / 2}[\mathrm{~ns}]\end{array}$ & $\begin{array}{c}\text { Quadru- } \\
\text { pole } \\
\text { moment [b] }\end{array}$ & $\begin{array}{c}E\left(\gamma_{1}\right) / E\left(\gamma_{2}\right) \\
{[\mathrm{keV}]}\end{array}$ & $A_{22}$ \\
\hline${ }^{44} \mathrm{Sc}$ & ${ }^{44} \mathrm{Ti}$ & $47 \mathrm{a}$ & 68 & 156 & \pm 0.21 & $78 / 68$ & +0.04 \\
${ }^{77} \mathrm{Se}$ & ${ }^{77} \mathrm{Br}$ & $57 \mathrm{~h}$ & 24 & 9.3 & $\pm 0.79[45]$ & $752 / 248$ & -0.45 \\
${ }^{99} \mathrm{Tc}$ & ${ }^{99} \mathrm{Mo}$ & $66 \mathrm{~h}$ & 181 & 3.6 & unknown & $740 / 181$ & +0.10 \\
${ }^{99} \mathrm{Ru}$ & ${ }^{99} \mathrm{Rh}$ & $15 \mathrm{~d}$ & 89 & 21 & +0.22 & $527 / 89$ & -0.22 \\
${ }^{100} \mathrm{Rh}$ & ${ }^{100} \mathrm{Pd}$ & $3.6 \mathrm{~d}$ & 75 & 214 & \pm 0.08 & $84 / 75$ & +0.16 \\
${ }^{111} \mathrm{Cd}$ & ${ }^{111} \mathrm{Ag}$ & $7.5 \mathrm{~d}$ & 245 & 85 & +0.83 & $96 / 245$ & -0.13 \\
${ }^{111} \mathrm{Cd}$ & ${ }^{111 \mathrm{~m}} \mathrm{Cd}$ & $48 \mathrm{~min}$ & 245 & 85 & +0.83 & $150 / 245$ & +0.16 \\
${ }^{111} \mathrm{Cd}$ & ${ }^{111} \mathrm{In}$ & $2.8 \mathrm{~d}$ & 245 & 85 & +0.83 & $171 / 245$ & -0.18 \\
${ }^{115} \mathrm{In}$ & ${ }^{115} \mathrm{Cd}$ & $53 \mathrm{~h}$ & 828 & 6 & \pm 0.61 & $35 / 492$ & +0.21 \\
${ }^{117} \mathrm{In}$ & ${ }^{117} \mathrm{Cd}$ & $2.4 \mathrm{~h}$ & 660 & 59 & \pm 0.60 & $89 / 354$ & -0.36 \\
${ }^{116} \mathrm{Sn}$ & ${ }^{116 \mathrm{~m}} \mathrm{Sb}$ & $60 \mathrm{~min}$ & 2365 & 370 & \pm 0.26 & $543 / 1072$ & +0.13 \\
${ }^{118} \mathrm{Sn}$ & ${ }^{118 \mathrm{~m}} \mathrm{Sb}$ & $5 \mathrm{~h}$ & 2321 & 22 & +0.16 & $254 / 1091$ & +0.11 \\
${ }^{129} \mathrm{I}$ & ${ }^{129 \mathrm{~m}} \mathrm{Te}$ & $69 \mathrm{~min}$ & 28 & 17 & -0.68 & $460 / 28$ & -0.05 \\
${ }^{133} \mathrm{Cs}$ & ${ }^{133} \mathrm{Ba}$ & $10.7 \mathrm{a}$ & 81 & 6 & -0.2 & $356 / 81$ & -0.04 \\
${ }^{140} \mathrm{Ce}$ & ${ }^{140} \mathrm{La}$ & $40.3 \mathrm{~h}$ & 2083 & 3.4 & +0.39 & $329 / 487$ & -0.13 \\
${ }^{172} \mathrm{Yb}$ & ${ }^{172} \mathrm{Lu}$ & $6.7 \mathrm{~d}$ & 1172 & 7.8 & +2.87 & $91 / 1094$ & +0.38 \\
${ }^{181} \mathrm{Ta}$ & ${ }^{181} \mathrm{Hf}$ & $42.4 \mathrm{~d}$ & 482 & 10.8 & +2.36 & $133 / 482$ & -0.29 \\
${ }^{187} \mathrm{Re}$ & ${ }^{187} \mathrm{~W}$ & $24 \mathrm{~h}$ & 206 & 563 & +3.04 & $479 / 72$ & -0.13 \\
${ }^{197} \mathrm{Hg}$ & ${ }^{197 \mathrm{~m}} \mathrm{Hg}$ & $24 \mathrm{~h}$ & 134 & 7 & \pm 0.49 & $165(\mathrm{e}-) / 134$ & +0.22 \\
${ }^{204} \mathrm{~Pb}$ & ${ }^{204 \mathrm{~m}} \mathrm{~Pb}$ & $67 \mathrm{~min}$ & 1274 & 270 & +0.44 & $912 / 375$ & +0.22 \\
${ }^{204} \mathrm{~Pb}$ & ${ }^{204} \mathrm{Bi}$ & $11.2 \mathrm{~h}$ & 1274 & 270 & +0.44 & $984 / 375$ & -0.05
\end{tabular}

short rise- and decay-time and a much slower one in the $360 \mathrm{~nm}$ region. If the UV component is used for the derivation of the time signal the obtainable time resolutions are in the range of 0.1-1 ns FWHM. At the same time by integrating the slow component an energy resolution of $10 \%$ can be achieved. If necessary it can even be improved by cooling the crystals moderately [11]. Additionally, $\mathrm{BaF}_{2}$ is not hygroscopic and can be machined into any form which makes the design of new, even more efficient PAC set-ups much easier [12, 13].

The radioactive probe nuclei necessary for a PAC experiment are either commercially available or can be produced by reactor or cyclotron irradiation, so that no detailed description of the production methods is necessary. A survey of the most common PAC probe nuclei and their relevant properties is given in the Table.

A much larger problem is usually the introduction of the probe nuclei into the material under investigation. Classical alloying or diffusion is only possible in 
a comparatively small number of cases, since a limited solubility, largely differing melting points, reactions of the small number of probe atoms with impurities in or above the melt cause numerous problems.

For this reason, the ion implantation technique, especially in semiconductors, has made an invaluable contribution to the development of the PAC method as a tool for materials research. Today suitable procedures have been developed to produce ion beams of the common radioactive probes with sufficient efficiency $[14,15]$ and naturally they can be implanted into any host material. Typical implantation energies range from 60 to $350 \mathrm{keV}$ which ensures in most of the probe-host combinations that the implanted probes are buried in the bulk of the host material.

The absolute number of probe nuclei necessary for a PAC experiment is very low $\left(10^{11}\right.$ atoms in the case of $\left.{ }^{111} \mathrm{In}\right)$ so that for example in alloyed samples the purity of the material is practically left unchanged. However, since in implanted samples the probe nuclei are concentrated in a relatively thin layer, locally the concentration can reach values of the order of 1 at. $\%$.

Finally, it should be mentioned that the final site of the implanted probe atom is generally determined by the elemental properties of the parent isotope, as one would intuitively expect. Therefore, in the case of defect investigations, one has always to keep in mind that not only the lattice site of the probe atom is selected by the elemental properties of the probe atom but also the trapping behaviour towards defects as well as the formation probability and stability of defect complexes is usually governed by the parent atoms.

Unfortunately, the ion implantation technique used in the sample preparation invariably leads to the production of damage in the sample. During the stopping process of the implanted heavy ions a large number of defects are created. Most of them recombine immediately in the collision cascade. However, a few percent survive and are located near the stopping site of the implanted atom. Therefore, this damage is usually called correlated damage. By varying the temperature at which the implantation takes place it is possible to control the type of defects retained in the sample, since all defects which are mobile during implantation will anneal immediately. Moreover, in order to investigate a specific defect, other methods of defect production like quenching (vacancies), electron irradiation (point defects), irradiation with light (p, $\alpha_{i}$ ) or heavy ions can be used. Since in this case the distribution of defects in the sample is not correlated to the previously implanted PAC probe atoms, it is called uncorrelated damage.

Implantation or diffusion of impurity atoms can be used to dope the sample with controlled amounts of impurities. On the other hand, the uncontrolled intake of impurities (usually gases from the residual gas atmosphere or other elements from support structures during heat treatments in vacuum) presents a major problem for the investigation of semiconductors.

After a sample has been doped with the PAC probes and the desired defects, the probability of finding a defect so close to a probe atom that it can produce a 
sizable QI is usually negligibly low. The fraction of probe atoms decorated with the defect under study can be increased if there is an attractive interaction between the probe and the defect. This attraction can be caused by elastic, electronic or chemical effects. For example, the elastic strain produced by an oversized probe in a lattice can at least partially be removed by trapping a vacancy on a next-nearest neighbour site and is therefore energetically favourable. Similarly a valence difference can lead to an attractive interaction. If the lattice temperature is increased then to a value where the defects become mobile, they start to diffuse through the lattice and are trapped at the probe atoms. At some higher annealing temperature, eventually the binding energy of the probe-defect complex is exceeded and it dissociates, thus reducing again the fraction of decorated probe atoms. This trapping and detrapping of defects is usually studied by performing an isochronous annealing treatment of the sample, i.e. it is heated from the measuring temperature $T$ (typically 4.2 or $293 \mathrm{~K}$ ) to an elevated annealing temperature $T$ for a fixed time (typically $10 \mathrm{~min}$ ) and then again a PAC measurement is performed at $T$. By recording the fraction of decorated probe atoms versus the annealing temperature $T_{\mathrm{a}}$ one can derive the temperatures where the defect becomes mobile and where the probe atom-defect complex breaks up again.

\section{Cavities in silicon}

With the increasing integration of implanted electronic circuits it has become more and more important to protect the active device regions from metal impurities. This can be achieved by introducing regions in the crystal which trap detrimental metal impurities before they can interact with dopants, for instance, during annealing steps necessary to remove implantation induced damage. Sometimes the backside of implanted wafers is used to this end, however, the necessary preparation steps do not integrate well into the normal production cycle. Therefore, the fact that the internal surfaces of cavities produced by high dose He implantation in silicon act as sinks for transition metal contaminants has attracted much attention [16, 17]. The formation of such He-induced cavities has been extensively studied with transmission electron microscopy (TEM) [17] and the gettering of metals with Rutherford backscattering spectroscopy (RBS) [16]. The PAC method is an ideal tool to get further information about the structure of these cavities and the gettering mechanism at very low levels of contaminants.

For this study overlapping profiles of He and radioactive ${ }^{111} \mathrm{In}$ were implanted in undoped (100) FZ-silicon wafers. Helium was implanted with $10 \mathrm{keV}$, leading to a mean range of $120 \mathrm{~nm}$ and a width (FWHM) of $110 \mathrm{~nm}$. To investigate the influence of the He-concentration doses of $0.6,2$, and $6 \times 10^{16}$ ions $/ \mathrm{cm}^{2}$ were implanted leading to peak local concentrations of $3.6,12$ or $36 \times 10^{20} \mathrm{He} / \mathrm{cm}^{3}$, respectively. ${ }^{111} \operatorname{In}\left({ }^{111} \mathrm{Cd}\right)$ was implanted with $160 \mathrm{keV}$ (mean range: $80 \mathrm{~nm}$, FWHM: $50 \mathrm{~nm}$ ). Typically doses of $0.2-2 \times 10^{13}$ ions $/ \mathrm{cm}^{2}$ corresponding to peak local concentrations of $0.32-3.2 \times 10^{18} \mathrm{In} / \mathrm{cm}^{3}$ were reached. 
PAC spectra were taken during an isochronous annealing programme between $500^{\circ} \mathrm{C}$ and $1100^{\circ} \mathrm{C}$ carried out in a graphite strip rapid thermal annealing (RTA) set-up under flowing nitrogen [18]. It yielded the following results: directly after the In implantation and after annealing at $500^{\circ} \mathrm{C}$ a fraction $f_{V}$ of ${ }^{111} \mathrm{In}$ probes show a QIF, which is known to be caused by an In-vacancy-complex [19] (Fig. 1). At $600^{\circ} \mathrm{C}$ this complex dissolves and the corresponding In-probes are left in undisturbed substitutional sites $\left(f_{S}\right)$. However, a fraction $f_{\mathrm{C}}$ of about $30 \%$ of the In probes exhibits a second unique QIF of $\nu_{Q}^{C}=411(5) \mathrm{MHz}(\eta=0.25(3))$ which can be associated with cavities already present at the end of the implantation process. From $600^{\circ} \mathrm{C}$ on $f_{C}$ grows continuously at the expense of the substitutional fraction $f_{S}$ and the width of the frequency distribution around $\nu_{Q}$ decreases. Raineri et al. [17] found that after annealing at $800^{\circ} \mathrm{C}$ the process of cavity formation is essentially finished. The continuing increase in $f_{C}$ therefore seems to indicate that at higher temperatures migrating cavities sweep up more and more previously substitutional In probes and collect them on their inner surfaces. The largest fraction of the ${ }^{111}$ In probes exhibiting $\nu_{Q}^{C}$ was found if the cavities are grown after the In has been implanted and annealed.

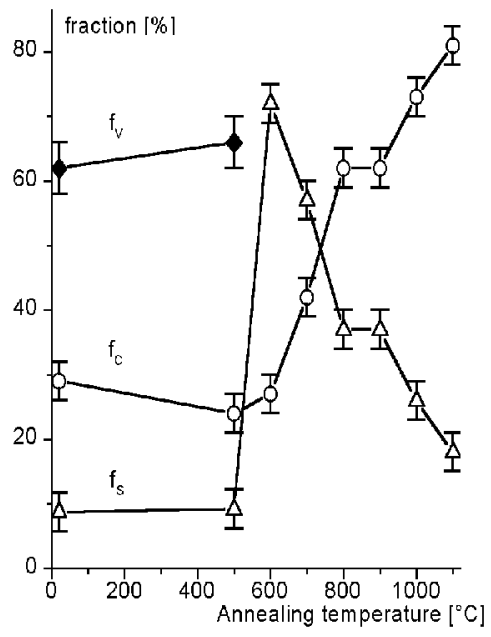

Fig. 1. Fractions of ${ }^{111}$ In probe atoms subjected to different electric field gradients as observed during an isochronal annealing programme, $f_{V}$ - In-vacancy complex, $f_{S}$ In in undisturbed substitutional sites, $f_{C}-$ In in cavities.

Reversing the order of cavity formation and ${ }^{111}$ In implantation leads only to a smaller fraction of In probes showing the characteristic QIF although, through the overlapping implantation profiles, ${ }^{111}$ In comes to rest in the same region where the cavities were formed.

Further, the influence of experimental parameters like the He dose, the im- 
plantation and annealing sequence and the orientation of the $\mathrm{Si}$ crystal relative to the detectors was studied [20]. All results support the assumption that the In probe atoms are situated in the inner walls of the cavities. Notably, the observed frequency $\nu_{Q}^{C}$ is quite similar but not equal to $\nu_{Q}=380(5) \mathrm{MHz}(\eta=0.23(3))$ a QIF measured for ${ }^{111}$ In on free $\operatorname{Si}(100)_{2 \times 1}$ surfaces [21]. However, in this case the QIF has been attributed to the formation of rows of In dimers on the Si surface.

In metals similar cavities are bounded by well-defined crystal surfaces and the In probes correspondingly showed the expected EFG orientation [22]. For Si Raineri et al. [17] found (100) and (111) planes as bounding surfaces of the cavities. However, for ${ }^{111} \mathrm{In}$ in the cavities the PAC spectra showed only very weak differences for different orientations. This behaviour is probably due to the relatively large number of possible orientations of the inner surfaces and is a subject to further investigations.

In conclusion, this example describing the interaction of ${ }^{111}$ In with He induced cavities in Si opens various possibilities to study the formation process of cavities as well as their effectiveness as a getter for metals, since it can be expected that an incorporation of metals into the cavities would change the QIF experienced by the In probe. Thus, an indirect study of the trapping process would become possible or, by means of a transition metal probe like ${ }^{100} \mathrm{Pd}\left({ }^{100} \mathrm{Rh}\right)$, for instance, the fate of impurities trapped in the cavities could be studied directly.

\section{Acceptor-donor pairs in Si under uniaxial stress}

In silicon, the interaction between donor atoms like $\mathrm{P}, \mathrm{As}$ and $\mathrm{Sb}$ and $\mathrm{In}$ acceptors has been studied by Wichert and Swanson [23]. They used ${ }^{111}$ In and could identify nearest neighbour pairs of $\mathrm{In}-\mathrm{P}_{1}, \mathrm{In}-\mathrm{A} \mathrm{s}_{1}$, and $\mathrm{In}-\mathrm{S} \mathrm{b}_{1}$ atoms characterized by their unique quadrupole interaction frequencies $\nu_{Q}=179(1) \mathrm{MHz}, 229(1) \mathrm{MHz}$, and 271(1) $\mathrm{MHz}$, respectively. In an effort to verify the structural models and the measured electric field gradient theoretical calculations were carried out based on the multiple scattering Korringa-Kohn-Rostoker (KKR) Green's function method [24] and, taking into account lattice relaxations around the complex, yielded values in good agreement with the experimental data. In view of these results it is interesting to study the behaviour of these complexes under mechanical uniaxial stress, i.e. to change the lattice constant in one major crystal direction.

Previously, PAC studies have shown that it is possible to produce quasi-homogeneous uniaxial stress at the site of probe nuclei near the surface by bending thin wafers of $\mathrm{Si}$ or $\mathrm{Ge}[25,26]$. The measurements indicated that at ${ }^{111} \mathrm{In}$ probes on substitutional sites in well-annealed Si sample tension induced QIF up to $38.5 \mathrm{MHz}$ can be induced by uniaxial tensile stress of $2.3 \mathrm{kbar}$ along the $\langle 110\rangle$ crystal axis.

Samples were prepared, $20 \mathrm{~mm}$ long and $7 \mathrm{~mm}$ wide, from a $100 \mu \mathrm{m}$ thick undoped Si wafer with $\langle 110\rangle$ surface orientation. The long edge of the samples was cut along a $\langle 110\rangle$ crystal axis, allowing for the possibility to exert stress in 
the surface layer along this axis by bending the sample. The bending radius could be adjusted by pressing the thin Si slab between the two heads of a micrometer screw. The maximum attainable relative change in lattice constant $\Delta L / L$ at the surface was calculated to be as large as $\sim 10^{-3}$.

First, the samples were implanted with ${ }^{111}$ In as an acceptor $(E=160 \mathrm{keV}$, $D=10^{12} \div 10^{13} \mathrm{In} / \mathrm{cm}^{2}$ ). Subsequently, the samples were annealed in an evacuated RTA set-up [18] at a temperature of $1173 \mathrm{~K}$ for $600 \mathrm{~s}$. This led to a full recovery of the radiation damage and the PAC spectra showed that all the implanted probe nuclei occupied substitutional lattice sites in the substrate lattice (Fig. 2). Applying an uniaxial stress of $1.8(1) \mathrm{kbar}$ along the $\langle 110\rangle$ crystal axis produced a tension induced frequency of $20(1) \mathrm{MHz}$ (Fig. 2), in good agreement with the literature [26].

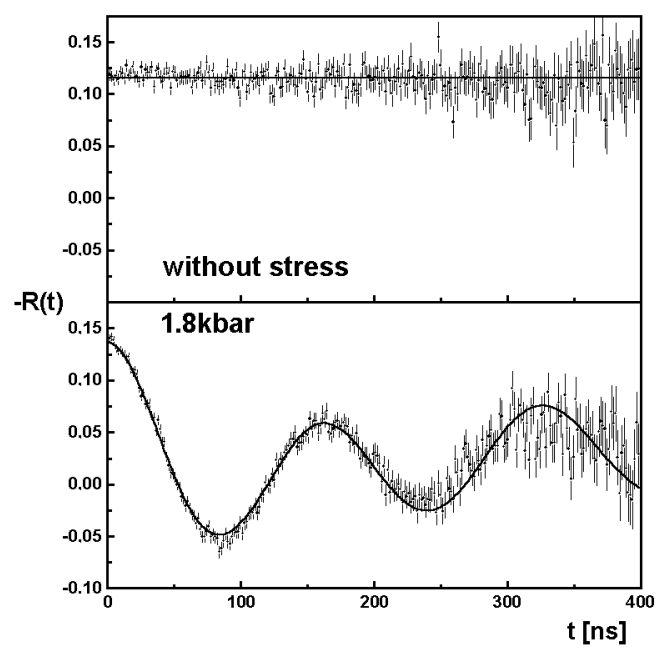

Fig. 2. PAC spectra from donor undoped sample showing the change of the anisotropy as the result of applied stress along $\langle 110\rangle$ crystal axis. The solid lines are theoretical fits.

Subsequently a donor impurity like phosphor $(E=60 \mathrm{keV}, D=2 \times$ $10^{14} \mathrm{P} / \mathrm{cm}^{2}$ ) was implanted to the same depth as the In probes and the sample annealed again at a temperature where a maximum fraction of acceptor-donor pairs are formed [23]. This occurs around $973 \mathrm{~K}$ for $\mathrm{P}$ doped samples. For a better recovery of the radiation damage of the samples, PAC measurements were only taken after a second annealing at $1173 \mathrm{~K}$ for $600 \mathrm{~s}$. The theoretical fit to the measured PAC data yielded for $25 \%$ of the In probes the expected QIF of $\nu_{Q}=179(1) \mathrm{MHz}$, corresponding to the formation of an In- $\mathrm{P}_{1}$ complex in Si.

Then the sample was bent, applying uniaxial stress in steps up to values of 1.8 kbar. Surprisingly, this produces no measurable effect on the QIF assigned to the In- $\mathrm{P}_{1}$ complex (Fig. 3). But also the QIF at the undisturbed substitutional site of the probe nuclei, which accounts for the larger fraction of probes in the 


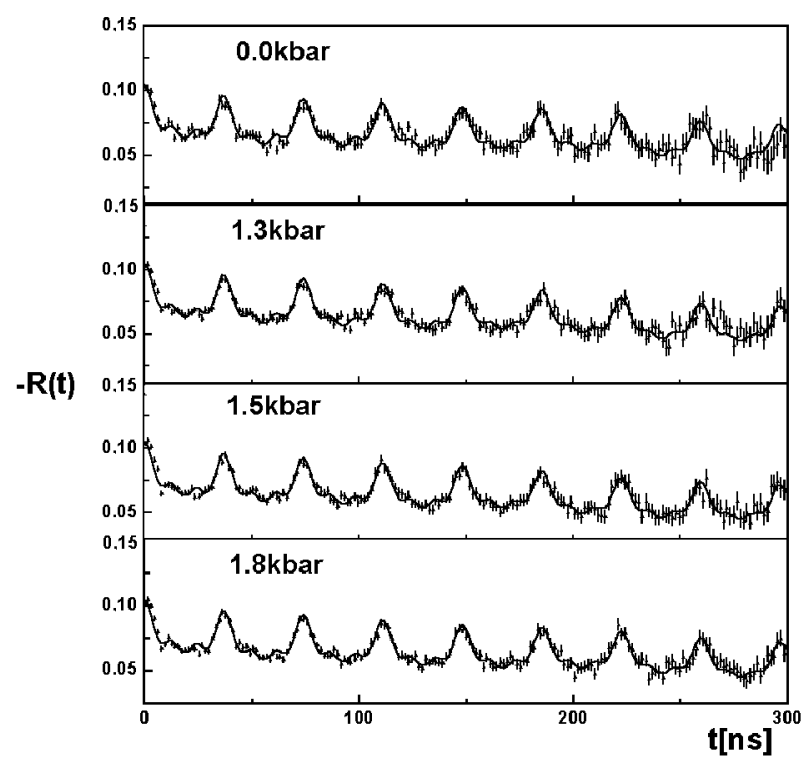

Fig. 3. PAC spectra for different values of applied uniaxial stress along the $\langle 110\rangle$ crystal axis (as indicated in the frames) for $\mathrm{P}$ doped samples. Measurements were taken with the $\langle 110\rangle$ crystal axis perpendicular to the detector plane.

sample, remains unchanged. Since the relative error of the QIF derived from a fit to the data is below $0.2 \%$, it can be deduced from the $r^{-3}$ dependence of the EFG that the relative lattice positions in the $\mathrm{In}_{-} \mathrm{P}_{1}$ complex change by less than $7 \times 10^{-4}$.

This unexpected result clearly indicates a different nature of the $\mathrm{P}$ implanted region as compared to the undoped samples. Under normal conditions a well-annealed sample would respond to the applied stress by producing a measurable QIF, which will vary with stress. Given the high sensitivity of the EFG to variations of the configuration of the complex, as estimated above, the shifts in the position of the lattice atoms surrounding the In probes must be negligible.

Therefore, we conclude that the implanted region has lost its elastic properties due to the $\mathrm{P}$ implantation. The best fit to the data from a $\mathrm{P}$ doped, unstressed sample yielded $15 \%$ of the probes in a non perfect cubic environment, experiencing nonunique EFGs due to lattice imperfections. This might be taken as an indication of the existence of a heterogeneous lattice structure in the region under study. Since usually dislocation and twinning effects are invoked to explain the inelastic behaviour of solids, this could indicate that the observed absence of stress in the implanted region is due to the presence of a large density of these extended defects. The argument is supported in the case of $\mathrm{P}$ implanted Si by TEM investigations of Prussin et al. [27] where, after annealing at $1173 \mathrm{~K}$ for $30 \mathrm{~min}$, a high density of dislocation loops is observed. 
It is interesting to note that in similar PAC investigations with As as a donor the uniaxial stress also produced no change of the QIF attributed to the $\mathrm{In}-\mathrm{As}$. pair. But there the In on undisturbed lattice sites showed the expected tension induced frequency. It is possible that the larger difference in the covalent radii between $\mathrm{P}$ and $\mathrm{Si}$ enhances the growth of dislocations loops during annealing. This effect is smaller in the case of As donors [28, 29] since its covalent radius is similar to that of Si. Therefore, we conclude that the observed absence of stress in the implanted region is due to inelastic deformation taking place due to the dense network of dislocation loops.

\section{Implantation in nitrides}

The group III - nitrides AIN, GaN, and $\operatorname{InN}$ are attractive materials for optoelectronic devices and especially GaN-based laser and light-emitting diodes are already commercialised on a large scale. However, presently available devices generally are produced by doping during growth [30] and not by ion implantation like most modern electronic devices. In order to couple such devices into integrated circuits, doping by ion implantation would be highly desirable. Besides the devices mentioned above, Er doped materials are of great interest for photonic applications such as solid state lasers, optical amplifiers, lasers storage devices and displays. The $\mathrm{Er}^{3+}$ intra $-4 f$ emission at $1.54 \mu \mathrm{m}$ corresponds to the standard wavelength used in telecommunication. Up to now Er doping of silicon has been intensively studied, but unfortunately the luminescence efficiency is strongly quenched at room temperature. In wide band gap semiconductors like GaN this quenching is much less pronounced [31] and thus it is hoped that Er doping by implantation might open the way to fully integrated optical circuits. Therefore, a profound knowledge of the annealing process, necessary to remove the implantation induced lattice damage and activate the implanted dopants, is essential. Only a few studies exist addressing this problem [32-34] where mainly electrical properties of the dopants were measured. Here we employ the PAC technique, using the probe ${ }^{181} \mathrm{Hf}\left({ }^{181} \mathrm{Ta}\right)$, since its elemental properties are similar to rare earths like Er and it is assumed that it will give information also relevant to these interesting implants.

The GaN samples used were grown by metal organic chemical vapour deposition (MOCVD) at $1040^{\circ} \mathrm{C}$ on $c$-plane sapphire substrates. The layers were nominally undoped $\left(n \sim 5 \times 10^{16} \mathrm{~cm}^{-3}\right)$ and were $\sim 3 \mu \mathrm{m}$ thick. The analysis by $\mathrm{X}$-ray diffraction and transmission electron microscopy showed them to be typical of current state-of-the-art heteroepitaxial material, with a rocking curve full-width-half-maximum (FWHM) of $\sim 280 \mathrm{arcsec}$ and defect densities of $\sim 10^{9} \mathrm{~cm}^{-2}$ at the surface.

The samples were cut to $5 \times 5 \mathrm{~mm}^{2}$ pieces and without further treatment implanted at room temperature at the Bonn isotope separator with ${ }^{181} \mathrm{Hf}$. It was produced by thermal neutron capture from the stable isotope ${ }^{180} \mathrm{Hf} .{ }^{*}$ The implan-

*The support of the GKSS-Forschungszentrum Geesthacht GmbH, Max-Planck-Straße, 
tation energy was $160 \mathrm{keV}$ and typical doses reached $10^{13} \mathrm{at} . / \mathrm{cm}^{2}$ mainly due to the line of stable ${ }^{180} \mathrm{Hf}$ which, due to the limited resolution of the isotope separator, overlaps the mass 181 line to a very small extent. A TRIM [35] calculation yielded a range of $314 \AA$ and a typical concentration of $\sim 4 \times 10^{18} \mathrm{Hf} / \mathrm{cm}^{-3}$.

Subsequently an isochronal annealing program was carried out in order to study the lattice damage recovery. The annealing steps were performed in a rapid thermal annealing apparatus [18] between graphite strips under a nitrogen atmosphere at ambient pressure. Additionally a second unimplanted piece of GaN was placed on the sample as a proximity cap to protect the surface. Typical holding times were $120 \mathrm{~s}$ and maximum temperatures of $1373 \mathrm{~K}$ were reached. Prior to the start of the programme and after each annealing step a PAC spectrum was taken.

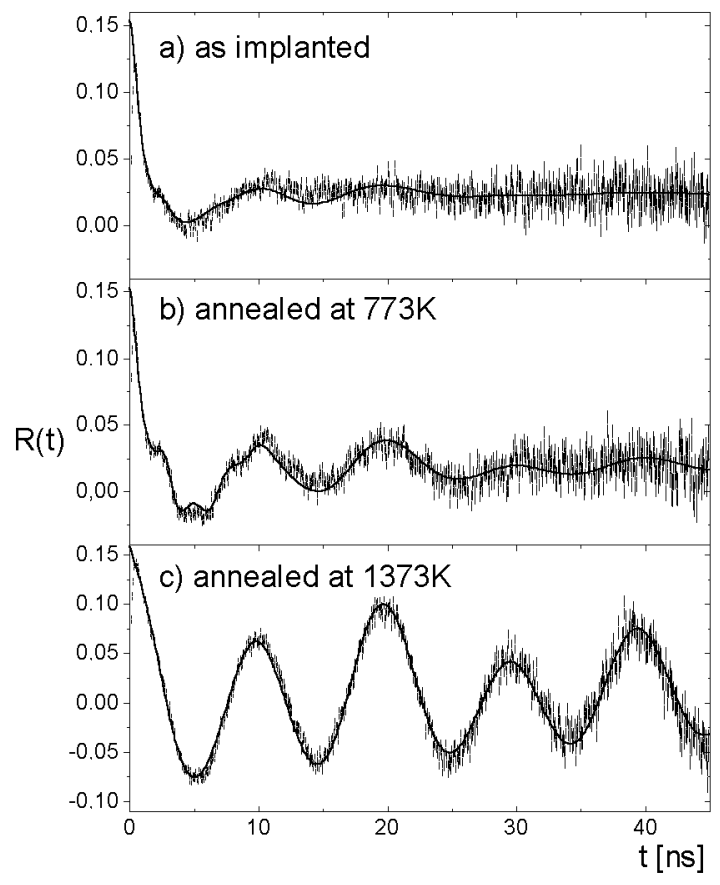

Fig. 4. PAC spectra observed for ${ }^{181} \mathrm{Hf}$ in GaN after annealing at different temperatures as indicated in the frames.

After the implantation, prior to any annealing, a damped oscillation was observed in the PAC spectra (Fig. 4a). This indicates that at the end of the collision cascade a small fraction $f_{S}$ of the implanted probe atoms stops on well-defined lattice sites. Least squares fits [36] to the data yield values for $f_{S}$ of $36 \%$ with a large frequency distribution of $\delta=20(1) \%$. The remaining fraction of the implanted ${ }^{181} \mathrm{Hf}$ probes $(64 \%)$ is situated in a lattice environment strongly

21502 Geesthacht, is gratefully acknowledged. 
disturbed by the implantation induced damage. They cause the sharp drop of the anisotropy in the first few nanoseconds in Fig. 4a and are characterised by a broad distribution of quadrupole interaction frequencies around $1000 \mathrm{MHz}$.

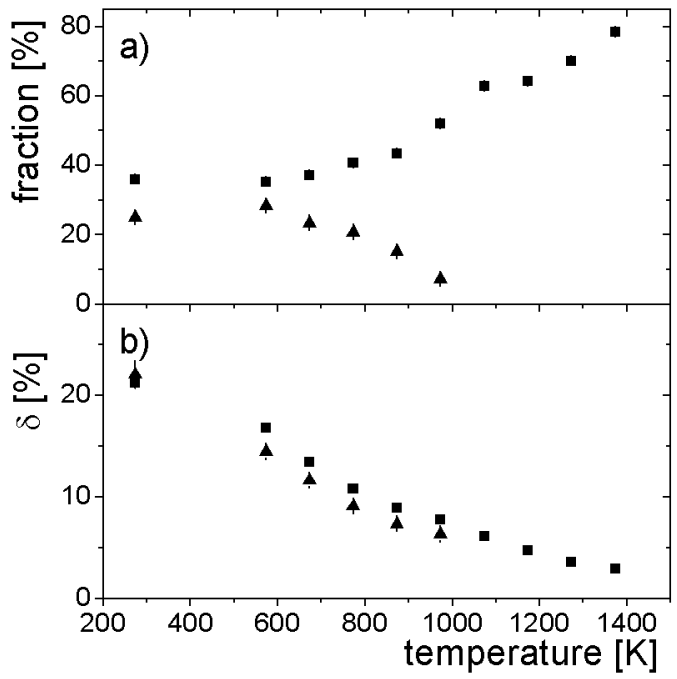

Fig. 5. (a) Fractions of implanted ${ }^{181} \mathrm{Hf}$ in GaN subjected to the electric field gradient at the substitutional Ga site ( $\boldsymbol{\square}$ ) and in the vicinity of a defect (full triangle) as observed during an isochronal annealing programme; (b) width $\delta$ of the corresponding quadrupole interaction frequencies.

Then, annealing causes an interesting development. First, up to a temperature of $T_{A}=673 \mathrm{~K}$, the damping parameter $\delta$ drops from $21 \%$ to $11 \%$ without a strong change in $f_{S}$ (see Fig. 5). Subsequently $\delta$ continues to decrease, although more slowly, whereas the fraction of probes on regular lattice sites starts to grow steadily until at $T_{A}=1373 \mathrm{~K}$ typically values of $f_{S}=70 \%$ to $80 \%$ are reached (Fig. 5). After the annealing step at $1373 \mathrm{~K}$ a quadrupole interaction constant of $\nu_{Q 1}=338(2) \mathrm{MHz}\left(\delta_{1}=3 \%, \eta_{1}=0\right)$ was derived.

The orientation of the corresponding EFG was checked by taking not only spectra with the sample's $\hat{c}$-axis oriented in different directions relative to the detectors. The results are in perfect agreement with the assumption that $V_{z z}$, the principal component of the EFG, is oriented parallel to the $\hat{c}$-axis of the wurtzite structure.

Since GaN crystallises in the hexagonal wurtzite structure an axially symmetric EFG with its principal component oriented along the $\hat{c}$-axis is expected at a regular lattice site. The observation of a unique EFG with this orientation indicates that the corresponding fraction $f_{S}$ of probe nuclei occupies regular sites. Due to the large difference in the covalent radii of $\mathrm{Hf}\left(r_{C}=1.44 \AA\right)$ and $\mathrm{N}$ $\left(r_{C}=0.75 \AA\right)$ the incorporation of $\mathrm{Hf}$ on $\mathrm{N}$ sites is very improbable and the incor- 
poration on Ga sites $\left(r_{C}=1.26 \AA\right)$ is more likely. This was confirmed by recent RBS measurements, which show that implanted Hf fully replaces Ga in GaN [37].

Dividing the measured QIF by the quadrupole moment $Q=2.36 \mathrm{~b}$ [38] of the $5 / 2$ intermediate state of the $\gamma-\gamma$ cascade in ${ }^{181} \mathrm{Ta}$ and the Sternheimer antishielding factor $\left(1-\gamma_{\infty}\right)=62[39]$ of the Ta ion the lattice EFG at the Ga site can be calculated to be $V_{Z Z}^{\text {latt }}=0.96 \times 10^{16} \mathrm{~V} / \mathrm{cm}^{2}$. This agrees well with the value of $V_{Z Z}^{\text {latt }}=0.65 \times 10^{16} \mathrm{~V} / \mathrm{cm}^{2}$ derived in the same way from NMR measurements for $\mathrm{Ga}$ in $\mathrm{GaN}$ [40], further confirming the complete substitutionality of the $\mathrm{Hf}$ probe.

At low annealing temperatures a second quadrupole interaction frequency $\nu_{Q 2}=1378(9) \mathrm{MHz}$ with an asymmetry parameter $\eta_{2}=0.63(2)$ is necessary to describe the spectra. A fraction of $f_{D}=25 \%$ of the probe atoms is subjected to the corresponding EFG (Fig. 4a). Immediately after implantation this EFG is highly non-unique resulting in large frequency distribution parameter $\delta_{2}=23 \%$, but $\delta_{2}$ drops parallel to $\delta_{1}$ (Fig. 5b) and after annealing at $773 \mathrm{~K}$ the frequency is clearly visible in the PAC spectrum (Fig. $4 \mathrm{~b}$ ). The fraction $f_{D}$ reaches a maximum of $28 \%$ at $573 \mathrm{~K}$ and disappears after annealing above $1000 \mathrm{~K}$ (Fig. 5a). The large value of $\nu_{Q 2}$ is characteristic for a deviation from the regular lattice structure in the nearest neighbourhood of the probe atoms. The low value of $\delta_{2}$ implies that this deviation has a regular structure, i.e. does not correspond to an amorphous environment. Similar situations have been observed in other semiconductors and are indicative of a unique defect trapped at the probe atom [41]. Since no diffusion in the Ga sublattice is expected in GaN below $1273 \mathrm{~K}$ [42], and the defect-probe atom complex breaks up below $1000 \mathrm{~K}$, we suggest that a nitrogen vacancy, trapped at the slightly oversized Hf probes, causes the corresponding quadrupole interaction. Estimates for a nearest neighbour vacancy in the point charge model [43] yield a magnitude of the observed EFG which is in good agreement with the experimental value. However, it seriously underestimates the lattice EFG at the Ga site and therefore cannot explain the large asymmetry parameter $\eta_{2}$.

Summarising, the PAC data lead to the following picture of the annealing process. For a fraction corresponding to the initial $f_{S}$ after the implantation no defect remains in the immediate vicinity of the probe atom. In this way, the lattice EFG is only slightly disturbed by more distant defects leading to the observed frequency distribution. Annealing leads to a partial recovery of these defects, thus narrowing $\delta_{1}$. Due to the larger mobility of nitrogen - nitrogen loss has been observed well below $1273 \mathrm{~K}$ [42] — these are most probably defects of the nitrogen sublattice. For the remaining fraction, defects in the nearest neighbourhood seriously disturb the lattice EFG. It takes temperatures well above $1000 \mathrm{~K}$, where also diffusion in the Ga sublattice sets in, to restore the surrounding lattice leading to a unique EFG at over $80 \%$ of the Hf probe sites. A small fraction of probes traps a nitrogen vacancy at a nearest neighbour site. The corresponding quadrupole interaction frequency also narrows until at $1000 \mathrm{~K}$ complete de-trapping occurs and 
the substitutional fraction is increased. Assuming first order exponential kinetics and de-trapping of the vacancy in a one step dissociation process, a binding energy $E_{B} \cong 3 \mathrm{eV}$ can be estimated. Here a Debye temperature of $586 \mathrm{~K}$ was used [44].

\section{Acknowledgment}

This work was partially supported by BMBF under contract No. MA06.06K.

\section{References}

[1] E.N. Kaufmann, R. Vianden, Rev. Mod. Phys. 51, 161 (1979).

[2] R. Vianden, NATO Advanced Studies Institute, Series E, Vol. 144, Kluwer, Dordrecht 1988, p. 239.

[3] Hyperfine Interaction of Defects in Semiconductors, Ed. G. Langouche, Elsevier, Amsterdam 1992.

[4] W. Pauli, Naturwissenschaften 12, 741 (1924).

[5] H. Kopfermann, Nuclear Moments, Academic Press, London 1958.

[6] E.A.C. Lucken, Nuclear Quadrupole Coupling Constants, Academic Press, London 1969 .

[7] Table of Isotopes, Eds. C.M. Lederer, V.S. Shirley, Wiley, New York 1978, App. VII: Table of nuclear moments.

[8] A. Lerf, T. Butz, Hyperfine Interact. 36, 275 (1987).

[9] A.R. Arends, C. Hohenemser, F. Pleiter, H. deWaard, L. Chow, R.M. Suter, Hyperfine Interact. 8, 191 (1980).

[10] M. Laval, M. Moszynski, R. Allemand, E. Comoreche, P. Guinet, R. Odru, J. Vacher, Nucl. Instrum. Methods 206, 109 (1983).

[11] K. Wisshak, K. Guber, F. Käppeler, Primärbericht 149191P20A, KFZ, Karlsruhe 1987.

[12] Th. Schäfer, Dissertation, University of Bonn, Bonn 1992.

[13] T. Butz, S. Saibene, Th. Fraenzke, M. Weber, Nucl. Instrum. Methods A 284, 417 (1989)

[14] Proc. of the 11th Int. Conf. on Electromagnetic Isotope Separators and Techniques Related to their Application, Ed. W.L. Talbert Jr., Nucl. Instrum. Methods B 26, (1987).

[15] H.L. Ravn, B.W. Allardyce, in: Treatise on Heavy Ion Science, Ed. D.A. Bromley, Vol. 8, Plenum Press, New York 1987, p. 363.

[16] S.M. Myers, G.A. Peterson, C.H. Seager, J. Appl. Phys. 80, 3717 (1996).

[17] V. Raineri, P.G. Fallica, G. Percolla, A. Battaglia, M. Barbagallo, S. Campisano, J. Appl. Phys. 78, 3727 (1995).

[18] G. Marx, Diploma Thesis, Rheinische Friedrich-Wilhelms-Universität Bonn, Bonn 1995.

[19] Th. Wichert, M. Deicher, G. Grübel, R. Keller, N. Schulz, H. Skudlik, Appl. Phys. A 48, 59 (1989). 
[20] J. Bartels, C. Noll, R. Vianden, Hyperfine Interct. 120/121, 353 (1999).

[21] G. Krausch, R. Fink, K. Jacobs, B. Luckscheiter, J. Lohmüller, B.-U. Runge, U. Wöhrmann, G. Schatz, Surf. Sci. 285, 81 (1993).

[22] R. Schumacher, R. Vianden, Phys. Rev. B 36, 8259 (1987).

[23] Th. Wichert, M.L. Swanson, J. Appl. Phys. 66, 3026 (1989).

[24] A. Settels, T. Korhonen, N. Papanikolaou, R. Zeller, P.H. Dederichs, Phys. Rev. Lett. 83, 4369 (1999).

[25] G. Marx, Dissertation, Bonn 1997.

[26] G. Marx, R. Vianden, Phys. Lett. A 210, 364 (1996).

[27] S. Prussin, K.S. Jones, Nucl. Instrum. Methods B 21, 496 (1987).

[28] K.S. Jones, S. Prussin, E.R. Weber, Nucl. Instrum. Methods B 21, 499 (1987).

[29] N. Smirnov, Sov. Phys. Dokl. 20, 224 (1975).

[30] S. Nakamura, T. Mukai, M. Senoh, Jpn. J. Appl. Phys. 30, L1998 (1991).

[31] U. Hommerich, M. Thaik, R.N. Schwartz, R.G. Wilson, J.M. Zavada, S.J. Pearton, C.R. Abernathy, J.D. MacKenzie, in: Proc. of the Symp. on Light Emitting Devices for Optoelectronic Applications and Twenty-Eighth State-of-the-Art Program on Compound Semiconductors, Electrochem. Soc., Pennington (NJ) 1998, p. 110.

[32] X.A. Cao, S.J. Pearton, R.K. Singh, C.R. Abernathy, J. Han, R.J. Shul, D.J. Rieger, J.C. Zolper, R.G. Wilson, M. Fu, J.A. Sekhar, H.J. Guo, S.J. Pennycook, MRS Internet J. Nitride Semicond. Res. 4S1, G6.33 (1999).

[33] H.H. Tan, J.S. Williams, J. Zou, D.J.H. Cockadyne, S.J. Pearton, R.A. Stall, Appl. Phys. Lett. 69, 2364 (1996).

[34] K. Lorenz, R. Vianden, S.J. Pearton, C.R. Abernathy, J. Zavada, MRS Internet J. Nitride Semicond. Res. 5, 5 (2000).

[35] J.P. Biersack, L.G. Haggmark, Nucl. Instrum. Methods 174, 257 (1980).

[36] N.P. Barradas, M. Rots, A.A. Melo, J.C. Soares, Phys. Rev. B 47, 8763 (1993).

[37] E. Alves, M.F. da Silva, J.G. Marques, J.C. Soares, K. Freitag, E-MRS 98, Mater. Sci. Eng. B 59, 207 (1999).

[38] T. Butz, A. Lerf, Phys. Lett. A 97, 217 (1983).

[39] F.D. Feiock, W.R. Johnson, Phys. Rev. 187, 39 (1969).

[40] G. Denninger, D. Reiser, Phys. Rev. B 55, 5073 (1997).

[41] T. Wichert, Characterization of Defects in Materials Symposium, Mater. Res. Soc., Pittsburgh (PA) 1987, p. 35.

[42] S. Strite, A. Pelzmann, T. Suski, M. Leszinynski, J. Jun, A. Rockett, M. Kamp, K.J. Ebeling, MRS Internet J. Nitride Semicond. Res. 2, 15 (1997).

[43] F.W. DeWette, G.E. Schacher, Phys. Rev. 137, A92 (1965).

[44] X.L. Chen, J.K. Liang, Y.P. Xu, P.Z. Jiang, Y.D. Yu, K.Q. Lu, Mod. Phys, Lett. $B$ 13, 285 (1999).

[45] H. Haas, D. Forkel-Wirth, P. Blaha, Hyperfine Interact. 120/21, 423 (1999). 